\title{
Structural basis for the molecular evolution of SRP-GTPase activation by protein
}

\author{
Gert Bange $^{1,3}$, Nico Kümmerer ${ }^{1,3}$, Przemyslaw Grudnik ${ }^{1}$, Robert Lindner ${ }^{1}$, Georg Petzold ${ }^{1,2}$, Dieter Kressler ${ }^{1,2}$, \\ Ed Hurt $^{1}$, Klemens Wild ${ }^{1} \&$ Irmgard Sinning $^{1}$
}

Small G proteins have key roles in signal transduction pathways. They are switched from the signaling 'on' to the non-signaling 'off' state when GTPase-activating proteins (GAPs) provide a catalytic residue. The ancient signal recognition particle (SRP)type GTPases form GTP-dependent homo- and heterodimers and deviate from the canonical switch paradigm in that no GAPs have been identified. Here we show that the YIxH protein activates the SRP-GTPase FlhF. The crystal structure of the Bacillus subtilis FlhF-effector complex revealed that the effector does not contribute a catalytic residue but positions the catalytic machinery already present in SRP-GTPases. We provide a general concept that might also apply to the RNA-driven activation of the universally conserved, co-translational protein-targeting machinery comprising the SRP-GTPases Ffh and FtsY. Our study exemplifies the evolutionary transition from RNA- to protein-driven activation in SRP-GTPases and suggests that the current view on SRP-mediated protein targeting is incomplete.

SIMIBI-class (named after the signal recognition particle, MinD, BioD) nucleotide-binding proteins appeared early in evolution ${ }^{1}$ and contain GTPases, as well as ATPases, involved in the correct localization of cellular constituents. The MinD ATPase, as the central part of the Min system, regulates the determination of the cell division site in all bacterial species ${ }^{2}$. SRP-GTPases form a subfamily of the SIMIBI class, with only three members: the signal sequence-binding protein Ffh (SRP54 in Eukarya and Archaea), the SRP receptor FtsY (SR $\alpha$ in Eukarya) and FlhF, which is involved in flagella biosynthesis ${ }^{3-5}$. They share the conserved NG domain, which contains two major additions to the conserved fold of small G proteins. First, an $\alpha-\beta-\alpha$ element (I-box) is inserted in the effector region; second, the $\mathrm{N}$ domain, comprising four $\alpha$-helices, is attached to the $\mathrm{N}$ terminus of the $\mathrm{G}$ domain. SRP (Ffh together with the SRP RNA) and FtsY constitute the universally conserved co-translational protein-targeting machinery ${ }^{6,7}$. When bound to GTP, Ffh and FtsY form, through interactions between their NG domains ${ }^{8,9}$, a heterodimeric complex that regulates the transfer of a ribosome-nascent chain complex to a vacant translocon in the membrane with a series of conformational rearrangements ${ }^{10,11}$. The two GTPases share a composite active site between their G domains in which GTP hydrolysis is reciprocally activated ${ }^{12}$. The SRP RNA ${ }^{13-15}$ and membrane lipids ${ }^{16,17}$ play fundamental roles in activating the Ffh-FtsY GTPases. The recent structure of the SRP-FtsY complex, together with biochemical implications, suggest that the distal end of the hairpin-like SRP RNA may be involved in this activation ${ }^{18}$. The third SRP-GTPase FlhF, together with the MinD-type protein YlxH (also known as FlhG, FleN, motR or MinD2), is essential for the placement and assembly of flagella ${ }^{19}$ in many polar and peritrichous flagellated bacteria ${ }^{20-24}$. FlhF is required for the targeting of the first flagellar protein, FliF, to the cell pole ${ }^{25}$ by a mechanism that is so far poorly understood. FlhF is associated with the membrane $e^{25,26}$ and localizes at the cell pole ${ }^{20}$. The FlhF protein (Fig. 1a) contains an $\mathrm{N}$-terminal B domain that seems to be involved in FliF targeting ${ }^{25}$; it shares the NG domain fold with the other two members of the SRP-GTPase subfamily. FlhF forms a stable homodimer with GTP and a composite active site that is basically identical to the active site of the Ffh-FtsY heterodimer ${ }^{5}$. In both the homo- and heterodimer, the two nucleotides are bound in a head-to-tail manner, with the $\gamma$-phosphate of one nucleotide interacting with the $3^{\prime}-\mathrm{OH}$ of the ribose moiety of the other. However, for the homo- and heterodimers formed by the three SRP-GTPases, the molecular mechanism of activation is still unknown. We set out to understand the activation of SRPGTPases by studying FlhF.

\section{RESULTS}

The SRP-GTPase FlhF is activated by YlxH

As FlhF (Fig. 1) forms a stable homodimer, and reciprocal activation has not been observed ${ }^{5}$, we reasoned that an external activator might exist. FlhF has been shown to co-immunoprecipitate with $\mathrm{YlxH}^{27}$, and both proteins directly interacted with each other in a yeast two-hybrid $(\mathrm{Y} 2 \mathrm{H})$ assay (Supplementary Fig. 1). The Y2H analysis also showed that the NG domain of FlhF (NG-FlhF) was sufficient for interaction with YlxH. As the dimerization of FlhF requires $\mathrm{GTP}^{5}$, we assessed a possible nucleotide dependence of the FlhF-YlxH interaction in vitro. Only in the presence of high concentrations of GTP and GMP-PNP could an interaction between both proteins be detected (Fig. 1b),

${ }^{1}$ Heidelberg University Biochemistry Center, Heidelberg, Germany. ${ }^{2}$ Present addresses: Research Institute of Molecular Pathology, Vienna, Austria (G.P.); Unit of Biochemistry, University of Fribourg, Fribourg, Switzerland (D.K.). ${ }^{3}$ These authors contributed equally to this work. Correspondence should be addressed to I.S. (irmi.sinning@bzh.uni-heidelberg) or G.B. (gert.bange@bzh.uni-heidelberg.de). 
Figure 1 The SRP-GTPase FlhF is activated by the MinD homolog $\mathrm{YIXH}$. (a) Domain structure of FIhF and $\mathrm{YIXH}$. The positions of the conserved nucleotidebinding elements (G1-G5) and the I-box in FIhF are indicated. YIxH contains an $\mathrm{N}$-terminal elongation (activator helix, $A H)$. The deviant walker A motif (DWA), switch regions (S1, S2), nucleotide specificity marker (NSM) and membrane targeting sequence (MTS), are indicated. (b) Coomassie-stained SDS-PAGE of NG-FIhF binding to immobilized GST-YIXH in the absence and presence of different nucleotides. The nucleotide concentration was $2.5 \mathrm{mM}$. Asterisks indicate NG-FIhF binding, confirmed by western blotting against its hexahistidine tag (lower panel). Lanes 1 and 2 show the purity of GST-YIXH and NG-FIhF. (c) YIxH enhances the activity of the FIhF GTPase. All measurements were done in triplicates. Error bars, s.d.

(d) Coomassie-stained SDS-PAGE of

NG-FIhF binding to immobilized GST-YIXH-N27 in the absence and presence of different nucleotides. Lanes 1 and 2 show the purity of GST-YIXHN27 and NG-FIhF. Asterisks indicate NG-FIhF binding, confirmed (lower panel) as described in b.
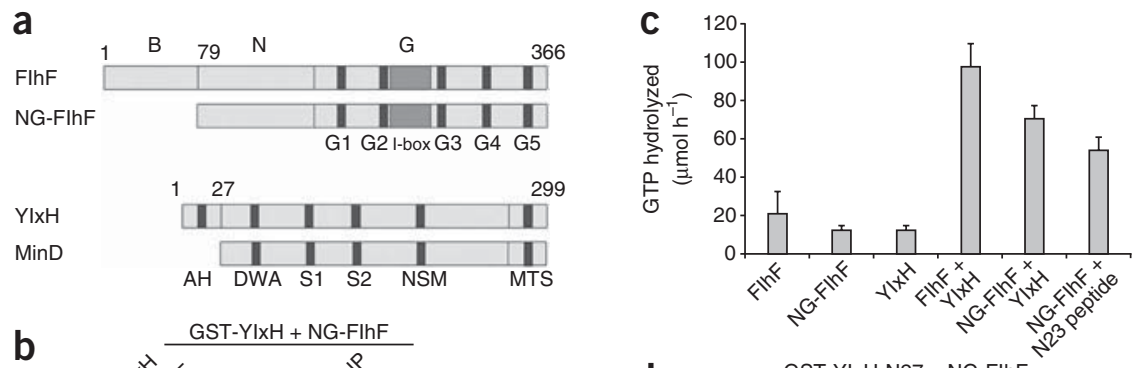

b

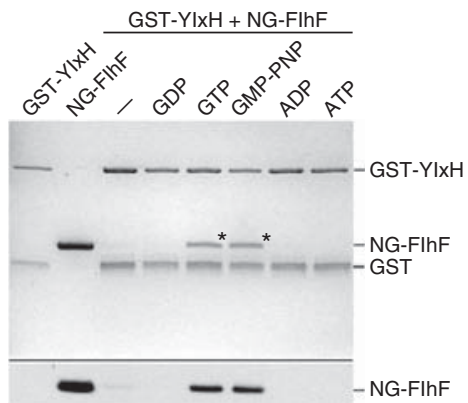

d

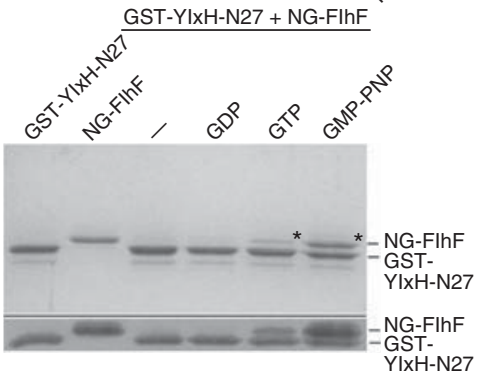

indicating that YlxH binds the GTP-bound dimer of FlhF. At low nucleotide concentrations, a stable association of both proteins was observed only in the presence of GMP-PNP, but not with GTP, indicating that YlxH stimulates the GTPase activity of FlhF (Supplementary Fig. 2). This notion is supported by a GTPase activity assay using GTP with traces of radioactively $\gamma_{-}{ }^{32} \mathrm{P}$-labeled GTP. The GTPase activities of FlhF and NG-FlhF were enhanced in the presence of YlxH, indicating that YlxH stimulates FlhF (Fig. 1c). To assess which part of $\mathrm{YlxH}$ is responsible for activation of FlhF, we compared it to its closest homolog MinD (Fig. 1a, Supplementary Fig. 3). Both proteins share a conserved ATPase domain. However, YlxH has a N-terminal extension that is absent from MinD. Deletion of this extension (YlxH- $\Delta \mathrm{N} 27)$ abolishes interaction of YlxH with NG-FlhF, in both the $\mathrm{Y} 2 \mathrm{H}$ assay and in vitro pulldown assay (Supplementary Fig. 4). Conversely, the $\mathrm{N}$ terminus of $\mathrm{YlxH}$ (N27) alone was sufficient to bind to FlhF in the presence of GTP or GMP-PNP (Fig. 1d) with a $K_{\mathrm{d}}$ of $0.6 \mu \mathrm{M}$ (Supplementary Fig. 5) and to stimulate the GTPase activity of FlhF (Figs. 1c). Therefore, the conserved N-terminal region of YlxH activates FlhF

\section{Crystal structure of the FlhF-effector complex}

To unravel the mechanism of how the N-terminal motif of YlxH stimulates the FlhF GTPase, we cocrystallized the NG domain of FlhF with a peptide containing the $\mathrm{N}$-terminal 23 residues of $\mathrm{YlxH}$ (YlxH-N23p). In order to mimic the transition state of hydrolysis, we added GDP and aluminum fluoride to the crystallization reaction ${ }^{28,29}$. The crystal structure (Table 1 and Fig. 2) showed that FlhF forms a symmetric homodimer, with the dimer interface exclusively established by the G domains. The two GTPase sites are paired to form a composite catalytic center in which two GDP- $\mathrm{AlF}_{3}-\mathrm{Mg}^{2+}$ ligands align in a head-to-tail manner.

During refinement, positive difference density was found in proximity to the I-box of each FlhF subunit and could be assigned unambiguously to residues Tyr6 to Glu21 of YlxH (Supplementary Fig. 6). The interaction of the N terminus of YlxH with FlhF accounts for $\sim 500 \AA^{2}$ of interface area and is established by hydrophobic and electrostatic contacts primarily with one FlhF subunit. Residues Ala9-Glu21 of YlxH form an $\alpha$-helix (activator helix) that binds to helices $\alpha \mathrm{G} 1$ (G domain) and

$\alpha 1$ (a (I-box) and to the G5 element (Fig. 2a,c; also see Fig. 3a). The conserved ${ }^{7}$ DQAXXLR motif (X, any residue; Fig. 2b) of YlxH is found at the $\mathrm{N}$ terminus of the activator helix, with DQA forming a $3_{10}$-helical turn that locates at the FlhF interface and contacts both catalytic half-sites. Most importantly, the side chain of Gln8 inserts into the composite active site at the interface of the two FlhF subunits and is clamped between the main chain of the G5 loop in cis and the G2 element

Table 1 Data collection and refinement statistics NG-FIhF/YIxH-N23p/GDP-AIF 3

\section{Data collection}

Space group

$P 1$

Cell dimensions

$$
\begin{aligned}
& a, b, c(\AA) \\
& \alpha, \beta, \gamma\left({ }^{\circ}\right)
\end{aligned}
$$

Resolution $(\AA)$

$R_{\mathrm{sym}}$

$I / \sigma I$

Completeness (\%)

Redundancy

$50.8,63.4,114.5$

$92.3,101.0,94.3$ $63.1-3.06(3.23-3.06)^{\mathrm{a}}$

$8.8(22.5)$

$8.5(5.0)$

$95.2(93.6)$

1.9 (1.9)

Refinement

Resolution ( $\AA$ )

No. of reflections

$63.1-3.06$

24,400

$R_{\text {work }} / R_{\text {free }}$

No. of atoms

$18.1 / 23.9$

Protein

9,010

8,712

Ligand/ion

207

Water

$B$-factors

Protein

Ligand/ion

Water

27.2

14.5

R.m.s. deviations

Bond lengths ( $\AA$ )

0.009

Bond angles $\left({ }^{\circ}\right)$

Values in parentheses are for the highest-resolution shell. 

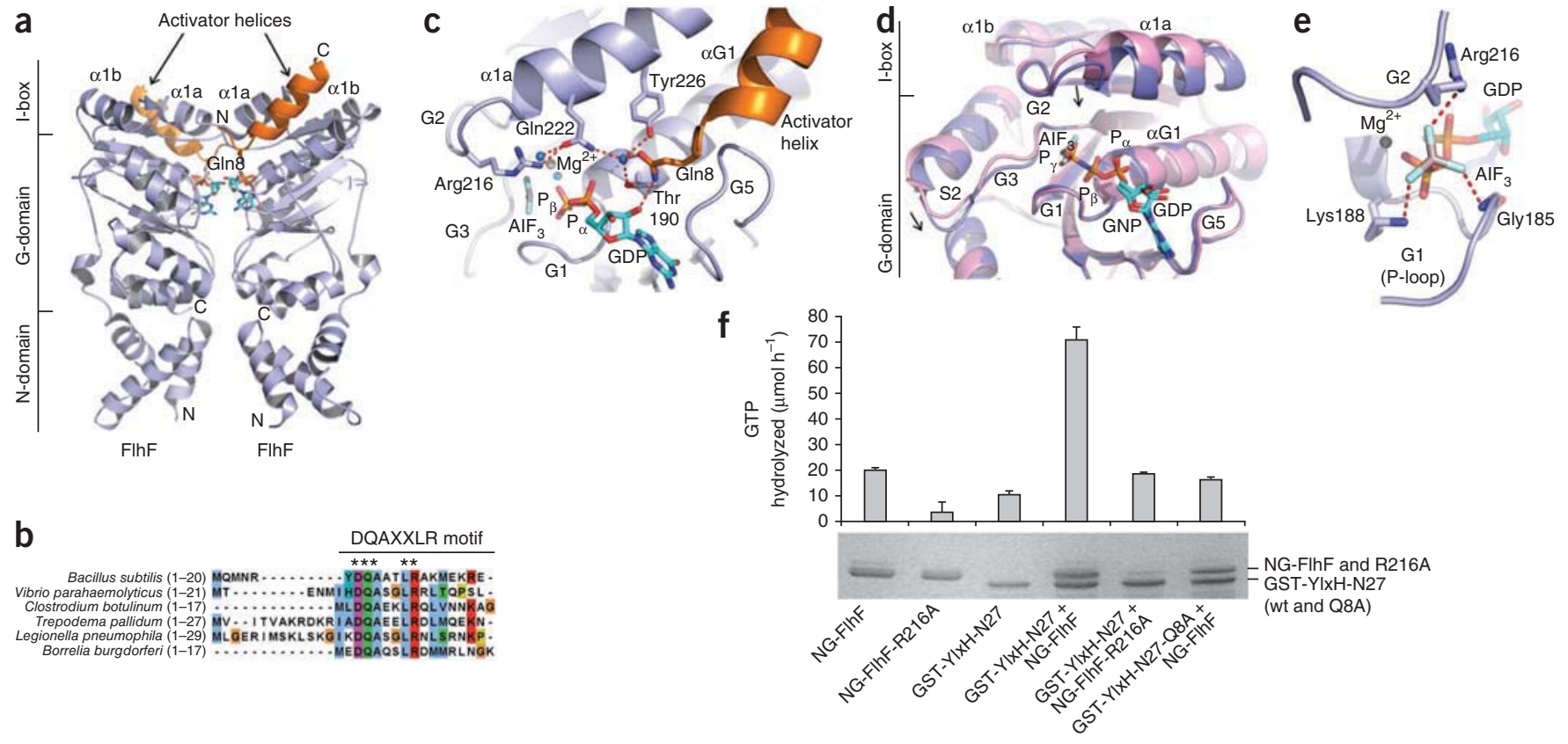

Figure 2 Structural basis for the activation of FIhF by YIxH. (a) Structure of the FIhF homodimer (blue) in complex with its activator (orange) and transition state mimic GDP-AIF 3 . The $\mathrm{N}$ and $\mathrm{C}$ termini are indicated by $\mathrm{N}$ and $\mathrm{C}$, respectively. (b) The DQAXXLR motif of YIxH is conserved among Gram-positive and Gram-negative bacteria. Strictly conserved residues are indicated by asterisks. (c) Key interactions in a catalytic half-site of the FlhF homodimer (blue). GIn8 of YlxH (orange) orchestrates the architecture of the composite active site to stabilize the catalytic conformation of Arg216. The G elements (G1-G3, G5) are shown. Red, dashed lines indicate possible hydrogen bond interactions (distance smaller than $2.9 \AA$ ); blue spheres

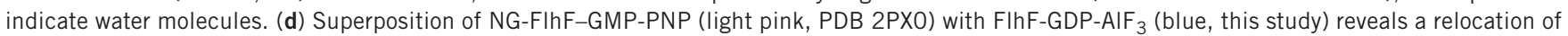
G2 as part of the I-box helix $\alpha 1$ a and the G3 element (indicated by arrows). (e) Stabilization of the transition state-mimicking aluminum fluoride (AIF ${ }_{3}$ ) by the G1 and G2 elements of FIhF. (f) Essential residues involved in enhancing the catalytic activity of FlhF. The catalytic and binding properties of different FIhF and YIXH variants were analyzed (upper panel). All measures were done in triplicate. Error bars, s.d. Coomassie-stained SDS-PAGE after an in vitro pulldown assay employing different NG-FIhF and YlxH-N27 variants (lower panel).

in trans (Fig. 2a; also see Fig. 3b, left side). Gln8 hydrogen bonding is complemented in cis to the 2' OH group of the ribose moiety of the nucleotide and to a buried water molecule, whose coordination is saturated by the interaction with Gln222 and Tyr226 from the I-box helix $\alpha 1$ and Thr190 from the $\alpha \mathrm{G} 1$ helix (Fig. 2c). The Gln8 interaction leads to a relocation of the G2 element, together with helix $\alpha 1$ a of the I-box and the G3 element, into the composite active site, when compared to the NG-FlhF-GMP-PNP structure ${ }^{5}$ (Fig. 2d). This relocation is the basis for the stabilization of the trigonal-bipyramidal transition state geometry. In particular, the G2-element Arg216, together with Gly185 and Lys 188 from the P-loop, perfectly triangulate the negative charges of the leaving group (mimicked by $\mathrm{AlF}_{3}$ ) (Fig. 2e), thus revealing the mechanism for the activation of GTP hydrolysis. Accordingly, replacement of Arg216 by alanine in NG-FlhF abolishes its binding to GST-YlxH-N27 (Fig. 2f). Mutation of Gln8 to alanine does not affect binding of the GST-YlxH-N27 to NG-FlhF ( $K_{\mathrm{d}} \approx 0.9 \mu \mathrm{M}$, Supplementary Fig. 5), but it completely abolishes activation, underlining its catalytic importance (Fig. 2f). This shows that the precise setup of the catalytic machinery of FlhF is a prerequisite for activation and illustrates the fine-tuning of effector binding to and activation of the GTPase.

\section{DISCUSSION}

\section{Mechanism of SRP-GTPase activation is conserved}

The FlhF-YlxH complex determines the mechanistic framework for the activation of an SRP-GTPase homodimer by protein. SRP-GTPase homo- and heterodimers contain the complete catalytic machinery in a composite active site $e^{5,8,9,30}$. Our structural and biochemical studies show that YlxH binding to FlhF leads to movement and stabilization of the G2 element and allows the correct placement of the catalytic arginine required for elevated GTP hydrolysis.

The homologous SRP-GTPases Ffh and FtsY form a heterodimer, which regulates co-translational protein targeting ${ }^{7}$. The recent crystal structure of SRP in complex with its receptor FtsY illustrates a dramatic conformational rearrangement in the SRP-FtsY complex ${ }^{18}$. The distal end of the SRP RNA, which is formed by the $5^{\prime}$ and $3^{\prime}$ ends of the molecule, locates at the G domain-I-box interface of the Ffh-FtsY heterodimer (Fig. 3a, right side). C83 of the SRP RNA approaches the catalytic chamber of the Ffh-FtsY heterodimer at a position similar to Gln8 of YlxH (Fig. 3a). The base of C83 seems to stabilize the G2 region of FtsY via a 'sandwich' involving Phe141, which corresponds to Tyr215 in FlhF (Fig. 3b). In a previous crystal structure of the Ffh-FtsY NG domain heterodimer containing GDP- $\mathrm{AlF}_{4^{-}}$, a peripheral nucleotide was found to bind in the same position as C83 (ref. 31), and mutation of C83 indeed affects SRPFtsY activity in vitro ${ }^{18}$. However, the low resolution of the SRP-FtsY structure and the absence of GDP-aluminum fluoride to mimic the transition state do not allow one to deduce the exact structural consequences of this RNA-GTPase contact for catalysis.

Given the high conservation of the composite active sites in the three SRP-GTPases, the mechanism of protein-driven activation of the FlhF homodimer might be directly translated to RNA-driven activation of the heterodimer. In both cases, interaction with the activator would induce the conformational stabilization of the G2 element required for efficient catalysis. Lowering entropy and enthalpy 
Figure 3 Mechanism and molecular evolution of SRP-GTPase activation. (a) Structure of the FlhF homodimer (blue) in complex with its activator (orange) and transition state mimic GDP-AIF 3 (left side). Structure of the FtsYFfh heterodimer (green) in complex with the regulatory, distal end of the SRP RNA (orange) and the nonhydrolyzable GTP analog GMP-PCP (guanosine- $5^{\prime}-[\beta, \gamma$-methyleno]triphosphate) (right side, PDB 2XXA, ref. 18). The M domain and helix $\alpha \mathrm{N} 1$ of the $\mathrm{N}$ domain of $\mathrm{Ffh}$ were excluded for clarity. (b) GIn8 of $\mathrm{YIXH}$ interacts with both FIhF subunits and clamps the G5-loop in cis with the G2 element in trans (left side). Nucleotide C83 of the SRP RNA interacts with FtsY and Ffh at the same regulatory regions (right side) as observed for GIn8 of YIxH for FIhF. (c) Top, activation of a small G protein (blue) by its GAP (gray). The GAP provides a catalytic residue (blue handle), which stabilizes the transition state (indicated by ' $\ddagger$ ') of the GTP (T) hydrolysis reaction. Bottom, activation of an SRP-GTPase dimer (blue). SRP-GTPases contain the complete catalytic machinery (kinked handle stick). However, activation requires the presence of the activator (orange, YIxH or SRP RNA), which positions the catalytic machinery for efficient stabilization of the transition state. (d) Molecular evolution of the FlhF-YlxH system. FlhF (blue) and YlxH (Iight pink) appeared by gene-duplication of Ffh (teal) and MinD (light pink), respectively. SRP RNA and activator helix are in orange. The question mark indicates a putative regulatory site.

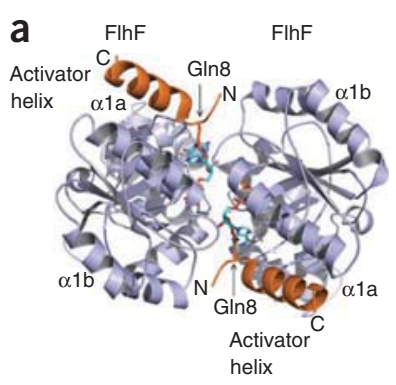

b

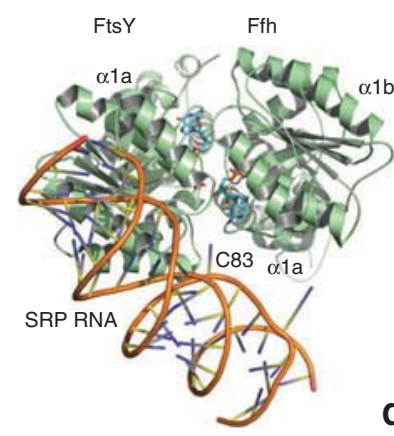

C

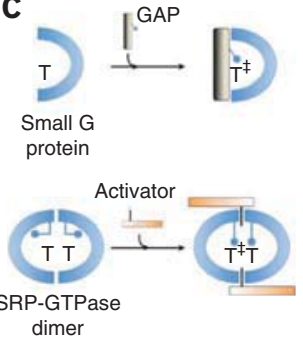

d
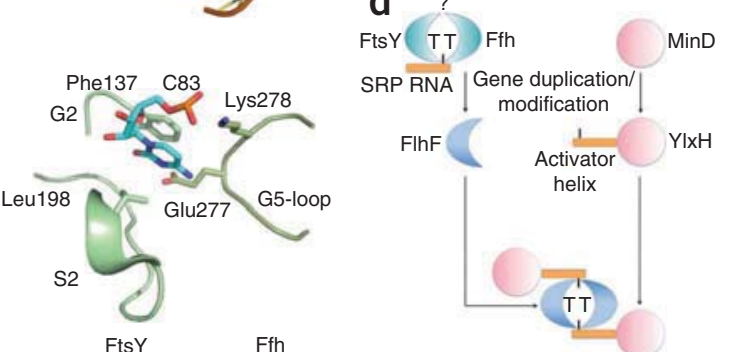

is a fundamental and ancient principle in enzyme catalysis ${ }^{32}$, and it is also applicable to SRP-GTPases. Therefore, the activation of SRPGTPase FlhF is different from activation of the well-studied canonical small GTPases ${ }^{33,34}$, where a GAP provides a catalytic residue in trans and thereby completes the catalytic center (Fig. 3c), for example, by an arginine or asparagine finger ${ }^{28,35}$. However, it is reminiscent of the activation of a monomeric $\mathrm{G} \alpha$ protein by its regulator protein RGS (regulator of $G$ protein signaling). The G $\alpha$ protein contains the complete set of catalytic residues, and RGS binding locks the flexible switch regions of $\mathrm{G} \alpha$ into their transition state conformations, promoting GTPase activity ${ }^{36}$. In particular, a conserved asparagine from RGS orients a catalytic glutamine in $G \alpha$ that is essential for transition state stabilization (Supplementary Fig. 7).

However, in the FlhF homodimer two YlxH molecules occupy identical binding sites, while in the nearly symmetric SRP-FtsY heterodimer, the presence of one RNA molecule adds to asymmetry. There might also be a second binding site present in the heterodimer (Fig. 3a,d) that could be used by other components of the targeting machinery to induce an additional level of regulation, for example, the ribosome or translocon. This component might have been overlooked because so far, the in vitro studies either were performed with only a subset of components or did not allow dissection of the individual contributions of components present.

\section{Evolution of SRP-GTPase activation by protein}

Mechanisms of protein targeting have been subject to strict evolutionary control, as the correct localization of macromolecular assemblies is of prime importance for all living cells. Our study defines the GTPase switch cycle of FlhF required for the spatial determination of the flagella biosynthesis site. Previous studies showed that FlhF and YlxH collaborate with each other to regulate flagella placement and number by a mechanism not yet understood ${ }^{27}$. We have now shown that binding of YlxH leads to the activation of FlhF and its inter-conversion into an inactive GDP-bound state. Indeed, functional studies show that the polar localization of FlhF, and thus of flagellum biosynthesis, is impaired by overexpression of YlxH in Vibrio cholerae ${ }^{27}$. This indicates that $\mathrm{YlxH}$ also acts as an activator of FlhF in vivo and therefore, might regulate the localization of FlhF at the cell pole.

Phylogenetic analysis shows that orthologs of $f l h F$ and $y l x H$ are present in $\sim 30 \%$ of all analyzed bacterial genomes and are widely distributed throughout all clades of bacterial phylogeny (Supplementary Fig. 8a). They always form a transcriptional unit with $y l x H$ being the adjacent downstream open reading frame of $f l h F$ (Supplementary Fig. 8a,b), which is remarkable considering the extensive rearrangements flagellar operons have undergone in evolution ${ }^{37}$. The absence of FlhF and YlxH in motile species most likely reflects the development of more recent regulatory systems, most notably in the $\alpha$-proteobacteria (see refs. 19,38 and Supplementary Fig. 8a), or a loss of polar flagellation in response to genome minimization, as indicated by a significant tendency $\left(P<10^{-5}\right)$ toward reduced genome and proteome size in such species (Supplementary Fig. 9). Agreement of phylogenetic distances inferred from the sequences of FlhF and $\mathrm{YlxH}$ with those of their putative ancestors $\mathrm{Ffh}$ and $\mathrm{MinD}^{1}$, respectively, as well as the more universal 16S rRNA distances (Supplementary Figs. 10 and 11) suggests that they form the core of a very ancient system for the spatial determination of the future flagellum site.

Likewise, SRP and FtsY form the regulatory core of the universally conserved, co-translational protein-targeting machinery. The presence of the highly conserved SRP RNA as a key regulatory element suggests a function of SRP that might reach as far back as the RNA world ${ }^{39,40}$. The MinD ATPase is conserved in all bacteria and plays a central role in determining the future cell division plane. Our study shows how bacteria used this well-established repertoire to develop new components and regulatory pathways (Fig. 3d). The modification of gene duplicates of $\mathrm{Ffh}$ and $\mathrm{MinD}^{1,39}$ into the homodimer-forming FlhF-GTPase and its activator YlxH, respectively, illustrates how evolution relies on existing structures and their combination into a new function. The replacement of the SRP RNA as an effector for SRP-GTPases by a protein parallels the transition from an ancestral RNA world into the modern protein world. 
Accession codes: Protein Data Bank. Atomic coordinates and structure factors for FlhF in complex with its activator have been deposited with accession code 3 SYN.

\section{ACKNOWLEDGMENTS}

This work was supported by the German Research Council SFB638 (I.S.), the Graduiertenkolleg GRK1188 and the interdisciplinary PhD program "Molecular Machines: Mechanisms and Functional Interconnections" of the Land BadenWürttemberg (I.S.). I.S. and E.H. are investigators of the Cluster of Excellence: CellNetworks. We are grateful to A. Hendricks for her excellent technical assistance, U. Pachmayr for her contribution in the beginning of the project and R. Pipkorn (Deutsches Krebsforschungszentrum) for peptide synthesis. We thank J. Kopp and C. Siegmann from the BZH/Cluster of Excellence: CellNetworks crystallization platform for their support and $\mathrm{E}$. Thomson for critical reading of the manuscript. Data collection was performed at ESRF beamline ID14-4 (European Synchrotron Radiation Facility).

\section{AUTHOR CONTRIBUTIONS}

G.B. and I.S. designed the experiments, analyzed the data and wrote the manuscript. G.B. and N.K. performed the experiments. D.K. and E.H. provided the yeast-two hybrid analysis. P.G. and G.P. contributed to the activation assays. G.B., K.W. and I.S. performed crystallographic analysis. R.L. performed the computational analysis. All authors commented on the manuscript.

\section{COMPETING FINANCIAL INTERESTS}

The authors declare no competing financial interests.

1. Leipe, D.D., Wolf, Y.I. Koonin, E.V. \& Aravind, L. Classification and evolution of P-loop GTPases and related ATPases. J. Mol. Biol. 317, 41-72 (2002).

2. Lutkenhaus, J. Assembly dynamics of the bacterial MinCDE system and spatia regulation of the Z ring. Annu. Rev. Biochem. 76, 539-562 (2007)

3. Montoya, G., Svensson, C., Luirink, J. \& Sinning, I. Crystal structure of the NG domain from the signal-recognition particle receptor FtsY. Nature 385, 365-368 (1997).

4. Freymann, D.M., Keenan, R.J., Stroud, R.M. \& Walter, P. Structure of the conserved GTPase domain of the signal recognition particle. Nature 385, 361-364 (1997).

5. Bange, G., Petzold, G., Wild, K., Parlitz, R.O. \& Sinning, I. The crystal structure of the third signal-recognition particle GTPase FlhF reveals a homodimer with bound GTP. Proc. Natl. Acad. Sci. USA 104, 13621-13625 (2007).

6. Grudnik, P., Bange, G. \& Sinning, I. Protein targeting by the signal recognition particle. Biol. Chem. 390, 775-782 (2009).

7. Cross, B.C., Sinning, I., Luirink, J. \& High, S. Delivering proteins for export from the cytosol. Nat. Rev. Mol. Cell Biol. 10, 255-264 (2009).

8. Egea, P.F. et al. Substrate twinning activates the signal recognition particle and its receptor. Nature 427, 215-221 (2004).

9. Focia, P.J., Shepotinovskaya, I.V., Seidler, J.A. \& Freymann, D.M. Heterodimeric GTPase core of the SRP targeting complex. Science 303, 373-377 (2004).

10. Zhang, X., Schaffitzel, C., Ban, N. \& Shan, S.O. Multiple conformational switches in a GTPase complex control co-translational protein targeting. Proc. Natl. Acad. Sci. USA 106, 1754-1759 (2009).

11. Wild, K., Halic, M., Sinning, I. \& Beckmann, R. SRP meets the ribosome Nat. Struct. Mol. Biol. 11, 1049-1053 (2004).
12. Powers, T. \& Walter, P. Reciprocal stimulation of GTP hydrolysis by two directly interacting GTPases. Science 269, 1422-1424 (1995).

13. Peluso, P., Shan, S.O., Nock, S., Herschlag, D. \& Walter, P. Role of SRP RNA in the GTPase cycles of Ffh and FtsY. Biochemistry 40, 15224-15233 (2001).

14. Siu, F.Y., Spanggord, R.J. \& Doudna, J.A. SRP RNA provides the physiologically essential GTPase activation function in cotranslational protein targeting. RNA 13, 240-250 (2007)

15. Bradshaw, N., Neher, S.B., Booth, D.S. \& Walter, P. Signal sequences activate the catalytic switch of SRP RNA. Science 323, 127-130 (2009).

16. de Leeuw, E. et al. Anionic phospholipids are involved in membrane association of FtsY and stimulate its GTPase activity. EMBO J. 19, 531-541 (2000).

17. Stjepanovic, G. et al. Lipids trigger a conformational switch that regulates signal recognition particle (SRP)-mediated protein targeting. J. Biol. Chem. 286, 23489-23497 (2011).

18. Ataide, S.F. et al. The crystal structure of the signal recognition particle in complex with its receptor. Science 331, 881-886 (2011).

19. Kirkpatrick, C.L. \& Viollier, P.H. Poles apart: prokaryotic polar organelles and their spatial regulation. Cold Spring Harb. Perspect. Biol. 3 (2011).

20. Murray, T.S. \& Kazmierczak, B.I. FlhF is required for swimming and swarming in Pseudomonas aeruginosa. J. Bacteriol. 188, 6995-7004 (2006).

21. Carpenter, P.B., Hanlon, D.W. \& Ordal, G.W. flhF, a Bacillus subtilis flagellar gene that encodes a putative GTP-binding protein. Mol. Microbiol. 6, 2705-2713 (1992).

22. Pandza, S. et al. The G-protein FlhF has a role in polar flagellar placement and general stress response induction in Pseudomonas putida. Mol. Microbiol. 36, 414-423 (2000)

23. Correa, N.E., Peng, F. \& Klose, K.E. Roles of the regulatory proteins FIhF and FIhG in the Vibrio cholerae flagellar transcription hierarchy. J. Bacteriol. 187, 6324-6332 (2005).

24. Kusumoto, A. et al. Regulation of polar flagellar number by the flhF and flhG genes in Vibrio alginolyticus. J. Biochem. 139, 113-121 (2006).

25. Green, J.C. et al. Recruitment of the earliest component of the bacterial flagellum to the old cell division pole by a membrane-associated signal recognition particle family GTP-binding protein. J. Mol. Biol. 391, 679-690 (2009).

26. Nowalk, A.J., Gilmore, R.D. Jr. \& Carroll, J.A. Serologic proteome analysis of Borrelia burgdorferi membrane-associated proteins. Infect. Immun. 74, 3864-3873 (2006).

27. Kusumoto, A. et al. Collaboration of FIhF and FlhG to regulate polar-flagella number and localization in Vibrio alginolyticus. Microbiology 154, 1390-1399 (2008).

28. Scheffzek, K. et al. The Ras-RasGAP complex: structural basis for GTPase activation and its loss in oncogenic Ras mutants. Science 277, 333-338 (1997).

29. Wittinghofer, A. Signaling mechanistics: aluminum fluoride for molecule of the year. Curr. Biol. 7, R682-R685 (1997).

30. Gasper, R., Meyer, S., Gotthardt, K., Sirajuddin, M. \& Wittinghofer, A. It takes two to tango: regulation of $\mathrm{G}$ proteins by dimerization. Nat. Rev. Mol. Cell Biol. 10, 423-429 (2009).

31. Focia, P.J., Gawronski-Salerno, J., Coon, J.S.T \& Freymann, D.M. Structure of a GDP:AIF4 complex of the SRP GTPases Ffh and FtsY, and identification of a peripheral nucleotide interaction site. J. Mol. Biol. 360, 631-643 (2006).

32. Fersht, A. Enzyme Structure and Mechanism (W.H. Freeman, San Francisco, 1977)

33. Bourne, H.R., Sanders, D.A. \& McCormick, F. The GTPase superfamily: a conserved switch for diverse cell functions. Nature 348, 125-132 (1990).

34. Vetter, I.R. \& Wittinghofer, A. The guanine nucleotide-binding switch in three dimensions. Science 294, 1299-1304 (2001).

35. Daumke, O., Weyand, M., Chakrabarti, P.P., Vetter, I.R \& Wittinghofer, A. The GTPase-activating protein Rap1GAP uses a catalytic asparagine. Nature 429, 197-201 (2004)

36. Kimple, A.J., Bosch, D.E., Giguere, P.M. \& Siderovski, D.P. Regulators of G-protein signaling and their $\mathrm{G} \alpha$ substrates: promises and challenges in their use as drug discovery targets. Pharmacol. Rev. 63, 728-749 (2011).

37. Liu, R. \& Ochman, H. Origins of flagellar gene operons and secondary flagellar systems. J. Bacteriol. 189, 7098-7104 (2007).

38. del Campo, A.M. et al. Chemotactic control of the two flagellar systems of Rhodobacter sphaeroides is mediated by different sets of CheY and FliM proteins. J. Bacteriol. 189, 8397-8401 (2007).

39. Bohnsack, M.T. \& Schleiff, E. The evolution of protein targeting and translocation systems. Biochim. Biophys. Acta 1803, 1115-1130 (2010).

40. Walter, P., Keenan, R. \& Schmitz, U. Perspectives: structural biology. SRP-where the RNA and membrane worlds meet. Science 287, 1212-1213 (2000). 


\section{ONLINE METHODS}

Yeast two-hybrid interaction analysis. Plasmids expressing the bait proteins, fused to the GAL4DNA-binding domain (G4BD), and the prey proteins, fused to the GAL4 activation domain (G4AD), were constructed in pGBKT7 (Clontech) and pGADT7 (Clontech), respectively, and combinations thereof were cotransformed into the reporter strain PJ69-4A (MATa trp1-901 leu2-3,112 ura3-

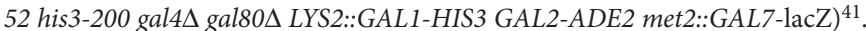
Yeast two-hybrid interactions were documented by spotting representative transformants in ten-fold serial dilution steps on SDC - Trp-Leu, SDC -Trp-Leu-His (HIS3 reporter) and SDC -Trp-Leu-Ade (ADE2 reporter) plates, which were incubated for $4 \mathrm{~d}$ at $30{ }^{\circ} \mathrm{C}$. As a positive control, the combination of plasmids pVA3-1 (expressing amino acids 72-390 of mouse p53, fused to G4BD, from pAS1 ${ }_{\mathrm{CYH} 2}$; Clontech) and pTD1-1 (expressing amino acids 87-708 of the SV40 large $\mathrm{T}$ antigen, fused to $\mathrm{G} 4 \mathrm{AD}$, from pACT2; Clontech) was used.

Expression and purification of recombinant proteins. FlhF and NG-FlhF were expressed and purified as previously described ${ }^{5}$. Briefly, proteins were expressed in Escherichia coli BL21(DE3) and purified by nickel-ion affinity and size exclusion chromatography (SEC). The SEC buffer was $20 \mathrm{mM}$ HEPES-Na, pH 7.5, $200 \mathrm{mM} \mathrm{NaCl}, 10 \mathrm{mM} \mathrm{KCl}$ and $10 \mathrm{mM} \mathrm{MgCl}_{2}$. The gene encoding $\mathrm{YlxH}$ was amplified by PCR from the Bacillus subtilis 168 genome and cloned into pET24d (Novagen) and pGAT2 (EMBL). YlxH contained an N-terminal hexahistidine tag and was expressed and purified as described for FlhF.

Peptide synthesis. The peptide synthesis unit of the German Cancer Research Center (DKFZ), Heidelberg, synthesized YlxH-N23p. The sequence was: ${ }^{1} \mathrm{MQ}$ MNRYDQAATLRAKMEKRERVL.

Crystallization, data collection, structure determination and analysis. Purified NG-FlhF was mixed with a two-fold excess of YlxH-N23p. Subsequently, $2 \mathrm{mM}$ GDP, $2 \mathrm{mM} \mathrm{AlCl}_{3}$ and $20 \mathrm{mM} \mathrm{NaF}$ were added. Prior to crystallization, the sample was incubated for $20 \mathrm{~min}$ at room temperature. Crystallization screens were performed at $291 \mathrm{~K}$ by the sitting-drop vapor-diffusion method. Equal volumes $(0.5 \mu \mathrm{l})$ of protein solution $\left(10 \mathrm{mg} \mathrm{ml}^{-1}\right)$ and crystallization buffer were mixed. The reservoir volume was $100 \mu$ l. Crystals appeared within $1-3 \mathrm{~d}$ in $0.1 \mathrm{M}$ Bis-Tris, $\mathrm{pH} 6.5$ and $1.8 \mathrm{M}$ ammonium sulfate. Diffraction data were measured on ID14-4 under cryogenic conditions (100 K, Oxford Cryosystems Cryostream) at the European Synchrotron Radiation Facility in Grenoble, France. Data were processed with iMosflm ${ }^{42}$. The crystal structure was determined by molecular replacement using Phaser ${ }^{42}$ and the crystal structure of NG-FlhF (PDB: 2PX3, ref. 5) as a search model. Model building and refinement were performed in Coot and PHENIX, respectively ${ }^{43,44}$. The values of the Ramachandran plot for the final refinement of the structure were: $94.7 \%$ preferred, $4.6 \%$ allowed and $0.7 \%$ outliers. Figures were prepared with PyMOL (http://pymol.org/).

Glutathione-S-transferase (GST) pulldown. All GST-fusion proteins used in this work contained an N-terminal GST-tag. The buffer used was PBS: $2 \mathrm{mM}$ $\mathrm{KH}_{2} \mathrm{PO}_{2}, 6.4 \mathrm{mM} \mathrm{Na}_{2} \mathrm{HPO}_{4}, 140 \mathrm{mM} \mathrm{NaCl}, 10 \mathrm{mM} \mathrm{KCl} .10 \mu \mathrm{l}$ of glutathioneSepharose beads (GE Healthcare) was incubated with $10 \mu \mathrm{g}$ of GST-YlxH and $400 \mu \mathrm{l}$ of PBS for $10 \mathrm{~min}$ on a spinning wheel at $4{ }^{\circ} \mathrm{C}$. Next, $40 \mu \mathrm{g}$ of NG-FlhF were added, and the volume was adjusted to $450 \mu \mathrm{l}$ with PBS. The binding reactions were incubated at $4{ }^{\circ} \mathrm{C}$ for $20 \mathrm{~min}$. Then the beads were washed three times with $400 \mu \mathrm{l}$ of PBS. Bound proteins were eluted with $40 \mu \mathrm{l}$ of $20 \mathrm{mM}$ glutathione, $20 \mathrm{mM}$ Tris- $\mathrm{HCl}, \mathrm{pH} 6.5$

Radioactive GTPase assay. GTPase activity was monitored by radioactive $\gamma^{32}$ P-labeled GTP as previously described ${ }^{16}$. The GTP hydrolysis reactions were performed in a total volume of $90 \mu \mathrm{l}$. The buffer was $20 \mathrm{mM}$ HEPES-Na, $\mathrm{pH} 7.5,200 \mathrm{mM} \mathrm{NaCl}, 10 \mathrm{mM} \mathrm{MgCl}_{2}$ and $10 \mathrm{mM} \mathrm{KCl}$. For the reaction, $0.5 \mathrm{mM}$ of guanosine $5^{\prime}$-triphosphate (GTP) and $16.5 \mathrm{nM} \gamma^{3}{ }^{32} \mathrm{P}$-labeled GTP was used. Reactions were incubated at room temperature. Reactions were stopped by addition of $90 \mu \mathrm{l}$ of $1 \mathrm{M}$ perchloric acid and neutralized with $30 \mu \mathrm{l}$ of $8 \mathrm{M}$ potassium acetate. Samples were analyzed by thin-layer chromatography and phosphorimaging. Intensities were calculated with ImageJ (http://rsb.info.nih.gov/ij/). The rate of hydrolysis (\%) was calculated as follows: hydrolysis $(\%)=\left[\operatorname{intensity~}\left({ }^{32} \mathrm{P}_{\mathrm{i}}\right)\right] /$ [intensity $\left({ }^{32} \mathrm{P}_{\mathrm{i}}\right)+$ intensity $\left.\left.\left(\gamma^{-}{ }^{32} \mathrm{P}-\mathrm{GDP}\right)\right]\right) \times 100$.

Determination of the dissociation constant $\left(K_{d}\right)$. GST-N27 was immobilized on GST-conjugated beads, and increasing amounts of NG-FlhF were added in the presence of $2.5 \mathrm{mM}$ of the nonhydrolyzable GTP analog GMP-PNP. SDSPAGE was carried out, and gels were stained with Coomassie Brilliant Blue. Band intensities were determined with ImageJ (http://rsb.info.nih.gov/ij/) and plotted against NG-FlhF concentration. The hyperbolic binding curve was fitted according to the equation $I=I_{\mathrm{Max}} \times[\mathrm{NG}-\mathrm{FlhF}] /\left(K_{\mathrm{d}}+[\mathrm{NG}-\mathrm{FlhF}]\right)$, where $I$ is intensity, $I_{\mathrm{Max}}$ is maximum intensity, [NG-FlhF] is concentration of NG-FlhF and $K_{\mathrm{d}}$ is the dissociation constant.

Discovery of $f l h F-y l x H$ orthologs. Phylogenetic analysis of the FlhF-YlxH system was based on the Kyoto Encyclopedia of Genes and Genomes (KEGG) ${ }^{45}$. Kegg Orthology (KO) groups K02404 and K04562 were used as a primer for the sequences of $F l h F$ and $Y l x H$, respectively. FlhF was unambiguously identified by its G1, G4 and G5 elements ${ }^{5}$. YlxH was defined by the conserved DQAXXLR motif (this study). These sequences were used to train a MEME model with discriminative conservation priors ${ }^{46}$ in order to generate sequence motifs that can discriminate FlhF from Ffh and FtsY, and YlxH from MinD. MAST search ${ }^{47}$ for these motifs in KEGG proteomes was carried out, followed by automated extraction of high-confidence hits $\left(E<10^{-6}\right)$ and revision of intermediateconfidence hits $(E<0.05)$, which contained the search motif within an expected range of positions.

Phylogenetic analysis and tree construction. Programs from the PHYLIP suite (http://evolution.genetics.washington.edu/phylip/) were applied for all phylogenetic analyses. Phylogenetic distances were inferred from ClustalW alignments (http://www.genome.jp/tools/clustalw/; ref. 48,) and tree topologies were constructed using neighbor joining. Reliability of the inferred topologies was assessed by comparison with 100 bootstrap replicates of each tree. Trees were drawn and juxtaposed using the APE package for R statistical software ${ }^{49}$.

41. James, P., Halladay, J. \& Craig, E.A. Genomic libraries and a host strain designed for highly efficient two-hybrid selection in yeast. Genetics 144, 1425-1436 (1996).

42. Collaborative Computational Project. N.. The CCP4 suite: programs for protein crystallography. Acta Crystallogr. D Biol. Crystallogr. 50, 760-763 (1994).

43. Emsley, P. \& Cowtan, K. Coot: model-building tools for molecular graphics. Acta Crystallogr. D Biol. Crystallogr. 60, 2126-2132 (2004).

44. Adams, P.D. et al. PHENIX: a comprehensive Python-based system for macromolecular structure solution. Acta Crystallogr. D Biol. Crystallogr. 66, 213-221 (2010).

45. Kanehisa, M., Goto, S., Furumichi, M., Tanabe, M. \& Hirakawa, M. KEGG for representation and analysis of molecular networks involving diseases and drugs. Nucleic Acids Res. 38, D355-D360 (2010).

46. Bailey, T.L., Boden, M., Whitington, T. \& Machanick, P. The value of position-specific priors in motif discovery using MEME. BMC Bioinformatics 11, 179 (2010).

47. Bailey, T.L. \& Gribskov, M. Combining evidence using p-values: application to sequence homology searches. Bioinformatics 14, 48-54 (1998).

48. Larkin, M.A. et al. Clustal $W$ and Clustal $X$ version 2.0. Bioinformatics 23, 2947-2948 (2007).

49. Paradis, E., Claude, J. \& Strimmer, K. APE: Analyses of phylogenetics and evolution in R language. Bioinformatics 20, 289-290 (2004). 